\title{
On Uniform Differentiability
}

by

\section{S. ROLEWICZ}

Presented by Czestaw OLECH

Summary. We introduce the notion of uniform Fréchet differentiability of mappings between Banach spaces, and we give some sufficient conditions for this property to hold.

Let $G$ be a convex set in an Asplund space $X$ (i.e. such that the dual $X_{0}^{*}$ to any separable subspace $X_{0} \subset X$ is separable) with non-empty interior. Let $f(\cdot)$ be a real-valued convex function defined on $G$. Asplund (1968) showed that in this case there is a dense $G_{\delta}$-set $\Omega \subset G$ such that the function $f(\cdot)$ is Fréchet differentiable at every point $x_{0} \in \Omega$. Moreover, on $\Omega$ the Fréchet derivative (gradient) is unique and it is continuous as a mapping from $\Omega$ into the conjugate space $X^{*}$, equipped with the norm topologies.

The result of Asplund (1968) can be extended to so called uniformly approximately convex functions (Rolewicz $(2002,2005)$ ).

Let $(X,\|\cdot\|)$ be a real Banach space. Let $f(\cdot)$ be a real-valued continuous function defined on an open convex subset $G \subset X$. We say that the function $f(\cdot)$ is uniformly approximately convex if for all $\varepsilon>0$ there is $\delta(\varepsilon)>0$ such that for all $x, y \in G$ such that $\|x-y\|<\delta$, and all $0 \leq t \leq 1$,

$$
f(t x+(1-t) y) \leq t f(x)+(1-t) f(y)+\varepsilon \min [t, 1-t]\|x-y\| .
$$

It can be shown that a function $f(\cdot)$ is uniformly approximately convex if and only if there is a non-decreasing function $\alpha(\cdot):[0,+\infty) \rightarrow[0,+\infty]$ such that

$$
\lim _{t \downarrow 0} \frac{\alpha(t)}{t}=0
$$

2000 Mathematics Subject Classification: Primary 46G05, 49J50.

Key words and phrases: uniformly Fréchet differentiable, uniformly approximately convex, subgradient, subdifferential. 
and

$$
f(t x+(1-t) y) \leq t f(x)+(1-t) f(y)+\min [t, 1-t] \alpha(\|x-y\|)
$$

(see Rolewicz (2001b)). The functions satisfying $\left(1_{\alpha}\right)$ for fixed $\alpha(\cdot)$ are called strongly $\alpha(\cdot)$-paraconvex.

The notion of uniformly approximately convex functions can be treated as a uniformization of the notion of approximately convex functions introduced in the papers of Luc, Ngai and Théra $(1999,2000)$. We recall that a realvalued function $f(\cdot)$ defined on a convex set $G \subset X$ is called approximately convex if for any $x_{0} \in G$ and $\varepsilon>0$ there is $\delta=\delta\left(\varepsilon, x_{0}\right)$ such that for $x, y$ such that $\left\|x-x_{0}\right\|<\delta$ and $\left\|y-x_{0}\right\|<\delta$ we have

$$
f(t x+(1-t) y) \leq t f(x)+(1-t) f(y)+\varepsilon \min [t, 1-t] .
$$

An essential role in extending Asplund's result to uniformly approximately convex functions is played by uniformly approximate subgradients. Let $f(\cdot)$ be a real-valued function defined on an open set $G$ of a Banach space $X$. We say that $f(\cdot)$ is uniformly approximately subdifferentiable if for all $\varepsilon>0$ there is $\delta(\varepsilon)>0$ such that for each $x \in G$ there is a linear functional $x^{*} \in X^{*}$ such that for $\|h\|<\delta$ we have

$$
f(x+h)-f(x)-x^{*}(h) \geq-\varepsilon\|h\| .
$$

The functional $x^{*}$ is called a uniformly approximate subgradient of $f(\cdot)$ at $x$. The set of all uniformly approximate subgradients of $f(\cdot)$ at $x$ will be called the uniformly approximate subdifferential of $f(\cdot)$ at $x$ and denoted by $\left.\partial^{u} f\right|_{x}$.

The notions of uniformly approximate subgradient and uniformly approximate subdifferential can be treated as a uniformization of the approximate subgradient and approximate subdifferential introduced by Mordukhovich and Ioffe (see Mordukhovich (1980, 1988), Ioffe (1984, 1986, 1989, 1990, 2000)).

Since we have the notions of uniformly approximate subgradients and uniformly approximate subdifferentials, it is a natural problem to introduce notions of uniformly approximate derivatives (gradients) and uniformly approximate differentials.

Let $G$ be an open subset of a Banach space $X$. Let $Y$ a Banach space. Let $\mathcal{F}(\cdot)$ be a mapping of $G$ into $Y$.

We say that the mapping $\mathcal{F}(\cdot)$ is uniformly Fréchet differentiable if for every $\varepsilon>0$ there is $\delta(\varepsilon)>0$ such that for each $x_{0} \in G$ there is a continuous linear operator $\left.\partial^{u} \mathcal{F}\right|_{x_{0}}(\cdot)$ mapping $X$ into $Y,\left.\partial^{u} \mathcal{F}\right|_{x_{0}}(\cdot) \in B(X \rightarrow Y)$, such that

$$
\left\|\mathcal{F}(x)-\mathcal{F}\left(x_{0}\right)-\left.\partial^{u} \mathcal{F}\right|_{x_{0}}\left(x-x_{0}\right)\right\|_{Y} \leq \varepsilon\left\|x-x_{0}\right\|_{X}
$$


provided $\left\|x-x_{0}\right\|_{X}<\delta$. The operator $\left.\partial^{u} \mathcal{F}\right|_{x_{0}}(\cdot) \in B(X \rightarrow Y)$ will be called a uniform Fréchet differential of $\mathcal{F}$ at $x_{0}$. It seems that this notion has not been considered even in finite-dimensional spaces.

Proposition 1. Let $G$ be an open set in a Banach space $X$. Let an equicontinuous mapping $\mathcal{F}(\cdot)$ from $G$ into a Banach space $Y$ be uniformly Fréchet differentiable. Then its uniform Fréchet differential $\left.\partial^{u} \mathcal{F}\right|_{x}$ is also equicontinuous in the norm operator topology with respect to $x$ on each subset $K \subset G$ such that

$$
d_{K}=\inf _{x \in K, y \notin G}\|x-y\|>0 .
$$

Proof. Let $K$ be as in the statement. Since the mapping $\mathcal{F}(\cdot)$ is uniformly Fréchet differentiable, for each $\varepsilon>0$ there is $r, 0<r<d_{K}$, such that for all $x \in K$ and all $h$ such that $\|h\| \leq r$ we have

$$
\left\|\mathcal{F}(x+h)-\mathcal{F}(x)-\left.\partial^{u} \mathcal{F}\right|_{x}(h)\right\|_{Y}<\varepsilon\|h\|_{X} .
$$

Since $\mathcal{F}(\cdot)$ is equicontinuous there is $\delta>0$ such that for all $x_{1}, x_{2} \in G$ such that $\left\|x_{1}-x_{2}\right\|_{X}<\delta$,

$$
\left\|\mathcal{F}\left(x_{1}\right)-\mathcal{F}\left(x_{2}\right)\right\|_{Y}<\varepsilon r .
$$

Since $r<d_{K}$, for $\|h\|_{X} \leq r$ and $x_{1}, x_{2} \in K$ we have

$$
\left\|\mathcal{F}\left(x_{1}+h\right)-\mathcal{F}\left(x_{2}+h\right)\right\|_{Y}<\varepsilon r .
$$

Take arbitrary $x_{1}, x_{2} \in K$ and arbitrary $h$ such that $\|h\|_{X}=r$. Thus by (6) and (7) we get

$$
\begin{aligned}
& \left\|\left.\partial^{u} \mathcal{F}\right|_{x_{1}}(h)-\left.\partial^{u} \mathcal{F}\right|_{x_{2}}(h)\right\|_{Y} \\
& \leq\left\|\left.\partial^{u} \mathcal{F}\right|_{x_{1}}(h)-\mathcal{F}\left(x_{1}+h\right)+\mathcal{F}\left(x_{1}\right)\right\|_{Y}+\left\|\mathcal{F}\left(x_{1}+h\right)-\mathcal{F}\left(x_{2}+h\right)\right\|_{Y} \\
& \quad+\left\|\mathcal{F}\left(x_{1}\right)-\mathcal{F}\left(x_{2}\right)\right\|_{Y}+\left\|\left.\partial^{u} \mathcal{F}\right|_{x_{2}}(h)-\mathcal{F}\left(x_{2}+h\right)+\mathcal{F}\left(x_{2}\right)\right\|_{Y} \\
& \leq 4 \varepsilon r .
\end{aligned}
$$

Since this holds for all $h$ such that $\|h\|_{X}=r$ we obtain

$$
\left\|\left.\partial^{u} \mathcal{F}\right|_{x_{1}}-\left.\partial^{u} \mathcal{F}\right|_{x_{2}}\right\|_{Y} \leq 4 \varepsilon .
$$

Therefore the gradient $\left.\partial^{u} \mathcal{F}\right|_{x}$ is equicontinuous on $K$.

There are two natural questions.

Problem 2. Is $\left.\partial^{u} \mathcal{F}\right|_{x}$ equicontinuous on the whole $G$ ?

Problem 3. Is the assumption that the mapping $\mathcal{F}(\cdot)$ is equicontinuous necessary?

It is easy to observe that there are non-equicontinuous functions with equicontinuous derivatives, which are uniformly Fréchet differentiable. 
ExAmple 4 . Let $X=\mathbb{R}$ and let $\mathcal{F}(x)=x^{2}$. It is easy to observe that the derivative $f^{\prime}(x)=2 x$ is equicontinuous but $\mathcal{F}(x)$ itself is not. The mapping $\mathcal{F}(\cdot)$ is uniformly Fréchet differentiable. Indeed,

$$
(x+h)^{2}-x^{2}-2 x h=h^{2} .
$$

It is a natural problem under which conditions a differentiable mapping $\mathcal{F}(\cdot)$ with equicontinuous derivative is uniformly Fréchet differentiable.

It is not difficult to give an example of a continuously differentiable mapping with equicontinuous derivative, which is not uniformly Fréchet differentiable.

ExAmple 5. Let $G$ be a spiral domain in $\mathbb{R}^{2}$ defined in the following way. Let $X=\{(r, D): 1-2 \pi / D<r<1-2 \pi /(D+1), 2 \pi<D<+\infty\} \subset \mathbb{R}^{2}$. Define $f: X \rightarrow \mathbb{R}^{2}$ by $f(r, D)=(r \cos D, r \sin D)$ and let $G=f(X)$. It is easy to see that $f: X \rightarrow G$ is one-to-one.

Define $\mathcal{F}: X \rightarrow \mathbb{R}$ by $\mathcal{F}(r, D)=D$. It is easy to see that the composite function $\mathcal{F} \circ f^{-1}: G \rightarrow \mathbb{R}$ is differentiable with equicontinuous derivative, but is not uniformly Fréchet differentiable.

However, we have

Proposition 6. Let $X, Y$ be Banach spaces. Let $G$ be a convex open set in $X$. Let $\mathcal{F}: G \rightarrow Y$ be differentiable and suppose that its derivative is equicontinuous. Then $\mathcal{F}(\cdot)$ is uniformly Fréchet differentiable.

Proof. If $x$ and $x+h$ belong to $G$ then

$$
\mathcal{F}(x+h)-\mathcal{F}(x)=\left.\int_{0}^{1} \partial^{u} \mathcal{F}\right|_{x+t h}(h) d t .
$$

Thus

$$
\mathcal{F}(x+h)-\mathcal{F}(x)-\left.\partial^{u} \mathcal{F}\right|_{x}(h)=\int_{0}^{1}\left[\left.\partial^{u} \mathcal{F}\right|_{x+t h}-\left.\partial^{u} \mathcal{F}\right|_{x}\right](h) d t .
$$

Since the derivative $\left.\partial^{u} \mathcal{F}\right|_{x}$ is equicontinuous with respect to $x$, for every $\varepsilon>0$ we can find $\delta>0$ such that if $\|h\|_{X}<\delta$ then $\left\|\left.\partial^{u} \mathcal{F}\right|_{x+t h}-\left.\partial^{u} \mathcal{F}\right|_{x}\right\|_{Y}<\varepsilon$ for all $0 \leq t \leq 1$. Therefore

$$
\left\|\mathcal{F}(x+h)-\mathcal{F}(x)-\left.\partial^{u} \mathcal{F}\right|_{x}(h)\right\|_{Y} \leq \int_{0}^{1}\left\|\left.\partial^{u} \mathcal{F}\right|_{x+t h}-\left.\partial^{u} \mathcal{F}\right|_{x}\right\|_{Y} d t\|h\|_{X}<\varepsilon\|h\|_{X} .
$$

The arbitrariness of $\varepsilon$ implies that $\mathcal{F}(\cdot)$ is uniformly Fréchet differentiable.

The convexity assumption in Proposition 6 can be weakened.

Let $G$ be an open set in a Banach space $X$. Let $K \geq 1$ be a constant. We say that $G$ is intervally $K$-convex if for any $x, y \in G$ there are elements $x=x_{0}, x_{1}, \ldots, x_{n}=y \in X$ such that $\left[x_{i-1}, x_{i}\right] \subset G$, where $[u, v]$ denotes the 
closed interval $\{t u+(1-t) v: 0 \leq t \leq 1\}$, and

$$
\sum_{i=1}^{n}\left\|x_{i-1}-x_{i}\right\|_{X} \leq K\|x-y\|_{X}
$$

(compare Rudnicki (1986)).

The set $G$ in Example 5 is not intervally $K$-convex for any $K \geq 1$.

Proposition 7. Let $X, Y$ be Banach spaces. Let $G$ be an intervally $K$ convex open set in $X$. Let $\mathcal{F}: G \rightarrow Y$ be differentiable and suppose that its derivative is equicontinuous. Then $\mathcal{F}(\cdot)$ is uniformly Fréchet differentiable.

Proof. Let $x \in G$ and let $h \in X$ be such that $y=x+h \in G$. Since $G$ is intervally $K$-convex, there are $x=x_{0}, x_{1}, \ldots, x_{n}=y=x+h$ such that $\left[x_{i-1}, x_{i}\right] \subset G$ and

$$
\sum_{i=1}^{n}\left\|x_{i-1}-x_{i}\right\|_{X} \leq K\|x-y\|_{X}
$$

Set $h_{i}=x_{i}-x_{i-1}$. Since $\left[x_{i-1}, x_{i}\right]=\left[x_{i-1}, x_{i-1}+h_{i}\right] \subset G$ we get

$$
\mathcal{F}\left(x_{i}\right)-\mathcal{F}\left(x_{i-1}\right)=\left.\int_{0}^{1} \partial^{u} \mathcal{F}\right|_{x_{i-1}+t h_{i}}\left(h_{i}\right) d t, \quad i=1, \ldots, n .
$$

Thus summing $\left(9_{i}\right)$ over $i$ we obtain

$$
\mathcal{F}(x+h)-\mathcal{F}(x)=\left.\sum_{i=1}^{n} \int_{0}^{1} \partial^{u} \mathcal{F}\right|_{x_{i-1}+t h_{i}}\left(h_{i}\right) d t .
$$

Observe that $h=\sum_{i=1}^{n} h_{i}$. Thus

$$
\left.\partial^{u} \mathcal{F}\right|_{x}(h)=\left.\sum_{i=1}^{n} \partial^{u} \mathcal{F}\right|_{x}\left(h_{i}\right)
$$

Subtracting (11) from (10) we get

$$
\mathcal{F}(x+h)-\mathcal{F}(x)-\left.\partial^{u} \mathcal{F}\right|_{x}(h)=\sum_{i=1}^{n} \int_{0}^{1}\left[\left.\partial^{u} \mathcal{F}\right|_{x_{i-1}+t h_{i}}-\left.\partial^{u} \mathcal{F}\right|_{x}\right]\left(h_{i}\right) d t .
$$

Since the derivative $\left.\partial^{u} \mathcal{F}\right|_{x}$ is equicontinuous with respect to $x$, for every $\varepsilon>0$ we can find $\delta>0$ such that if $\|x-z\|_{X}<K \delta$ then $\left\|\left.\partial^{u} \mathcal{F}\right|_{z}-\left.\partial^{u} \mathcal{F}\right|_{x}\right\|_{Y}<\varepsilon$. Since $G$ is an intervally $K$-convex open set, $\left\|x_{i-1}+t h_{i}-x\right\|_{X} \leq K\|h\|_{X}$.

Therefore if $\|h\|_{X}<\delta$ then

$$
\begin{aligned}
\| \mathcal{F}(x+h)-\mathcal{F}(x)- & \left.\partial^{u} \mathcal{F}\right|_{x}(h) \|_{Y} \\
& \leq \sum_{i=1}^{n} \int_{0}^{1}\left\|\left[\left.\partial^{u} \mathcal{F}\right|_{x_{i-1}+t h_{i}}-\left.\partial^{u} \mathcal{F}\right|_{x}\right]\right\|_{Y} d t\left\|h_{i}\right\|_{X} \\
& <\varepsilon \sum_{i=1}^{n}\left\|h_{i}\right\|_{X} \leq K \varepsilon\|h\|_{X} .
\end{aligned}
$$


The arbitrariness of $\varepsilon$ implies that the mapping $\mathcal{F}(\cdot)$ is uniformly Fréchet differentiable.

It is not clear if the condition that $G$ is an intervally $K$-convex open set is also necessary for the conclusion of Proposition 7 to hold. More precisely, we have

Problem 8. Suppose that $G$ is an open set in a Banach space $X$, which is not intervally $K$-convex. Can we construct a real-valued differentiable function $\mathcal{F}(\cdot)$ on $G$ with equicontinuous derivative, but not uniformly Fréchet differentiable?

\section{References}

E. Asplund (1966), Farthest points in reflexive locally uniformly rotund Banach spaces, Israel J. Math. 4, 213-216.

E. Asplund (1968), Fréchet differentiability of convex functions, Acta Math. 121, 31-47.

A. D. Ioffe (1984), Approximate subdifferentials and applications I, Trans. Amer. Math. Soc. 281, 389-416.

A. D. Ioffe (1986), Approximate subdifferentials and applications II, Mathematika 33, 111128.

A. D. Ioffe (1989), Approximate subdifferentials and applications III, ibid. 36, 1-38.

A. D. Ioffe (1990), Proximal analysis and approximate subdifferentials, J. London Math. Soc. $41,175-192$.

A. D. Ioffe (2000), Metric regularity and subdifferential calculus, Uspekhi Mat. Nauk 55, no. 3, 104-162 (in Russian).

D. T. Luc, H. V. Ngai and M. Théra (1999), On $\varepsilon$-monotonicity and $\varepsilon$-convexity, in: Calculus of Variations and Differential Equations, A. Ioffe et al. (eds.), Chapman \& Hall/CRC, Res. Notes Math. 410, Chapman and Hall, 82-100.

D. T. Luc, H. V. Ngai and M. Théra (2000), Approximate convex functions, J. Nonlinear Convex Anal. 1, 155-176.

B. S. Mordukhovich (1980), Metric approximations and necessary optimality conditions for general classes of nonsmooth extremal problems, Soviet Math. Dokl. 22, 526-530.

B. S. Mordukhovich (1988), Approximation Methods in Problems of Optimization and Control, Nauka, Moscow (in Russian).

S. Rolewicz (1979a), On paraconvex multifunctions, in: Oper. Research Verfahren 31, 540546.

S. Rolewicz (1979b), On $\gamma$-paraconvex multifunctions, Math. Japonica 24, 293-300.

S. Rolewicz (2000), On $\alpha(\cdot)$-paraconvex and strongly $\alpha(\cdot)$-paraconvex functions, Control Cybernet. 29, 367-377.

S. Rolewicz (2001a), On equivalence of Clarke, Dini, $\alpha(\cdot)$-subgradients and local $\alpha(\cdot)$ subgradients for strongly $\alpha(\cdot)$-paraconvex functions, Optimization 50, 353-360.

S. Rolewicz (2001b), On uniformly approximate convex and strongly $\alpha(\cdot)$-paraconvex functions, Control Cybernet. 30, 323-330.

S. Rolewicz (2002), $\alpha(\cdot)$-monotone multifunctions and differentiability of strongly $\alpha(\cdot)$ paraconvex functions, ibid. 31, 601-619.

S. Rolewicz (2005), On differentiability of strongly $\alpha(\cdot)$-paraconvex functions in nonseparable Asplund spaces, Studia Math. 167, 235-244. 
R. Rudnicki (1986), Asymptotic properties of the iterates of positive operators on $C(X)$, Bull. Polish Acad. Sci. Math. 34, 181-187.

S. Rolewicz

Institute of Mathematics

Polish Academy of Sciences

Śniadeckich 8, P.O. Box 21

00-956 Warszawa, Poland

E-mail: rolewicz@impan.gov.pl

Received May 4, 2008;

received in final form September 4, 2008 